\title{
LA REVISTA BROTERIA, LOS JESUITAS NATURALISTAS Y GONÇALO SAMPAIO. INTERCAMBIO DE PLANTAS E IDEAS Y EL DESARROLLO DE LA BOTÁNICA EN PORTUGAL
}

\author{
João Paulo Cabral*
}

Universidad de Oporto

\begin{abstract}
RESUMEN
La revista Broteria recorrió un largo camino, desde su fundación en 1902, hasta mediados de la década de 1920, cuando se consolida como una de las mejores revistas de historia natural y una voz de la renovación de las Ciencias Naturales en Portugal. El éxito de Broteria se debió, principalmente, a las notables calidades de sus fundadores y principales redactores: capacidad de trabajo, nivel intelectual y perseverancia; capacidad para establecer una red de naturalistas que les enviaban colecciones biológicas de regiones mal conocidas; capacidad de adaptación en el exilio continuando su trabajo y enfocando sus estudios en la historia natural del país de exilio. La manutención, en regular funcionamiento, de sus colegios y la apertura a la colaboración de naturalistas no jesuitas, especialmente a los botánicos de Oporto, también contribuyeron al éxito de Broteria. Los documentos epistolares tratados en este trabajo demuestran que A. Luisier, J.S. Tavares, y G. Sampaio lideraron una red de jesuitas naturalistas y botánicos de Oporto, que intercambiaron plantas e ideas. Este intercambio fue determinante en el estudio de la flora de musgos y plantas vasculares portuguesas y el progreso de los Herbarios de los colegios jesuitas y de la Academia Politécnica de Oporto.
\end{abstract}

PALABRAS CLAVE: Broteria. Gonçalo Sampaio. Botánicos jesuitas. Flora. Portugal.

THE JOURNAL BROTERIA, JESUIT BOTANISTS AND GONÇALO SAMPAIO. EXCHANGE OF PLANTS AND IDEAS, AND THE DEVELOPMENT OF BOTANY IN PORTUGAL

\section{ABSTRACT}

The journal Broteria has covered a long path, since its foundation in 1902 until the mid 20's, when it stands as one of the best journals of natural history and a voice of the renewal of the natural

* Profesor de la Facultad de Ciencias de la Universidad de Oporto e Investigador del Centro Interdisciplinar de Investigación Marina y Ambiental de la Universidad de Oporto. Email:.jpcabral@fc.up.pt. 
sciences in Portugal. Broteria's success was due, mainly, to the remarkable qualities of its founders and main editors: their working capacity, intellectual standards and perseverance as well as the ability to establish a network of naturalists who sent them biological collections from remote regions and the ability to adapt to exile, while continuing to work and focusing their studies on the natural history of the exile country. The maintenance, in regular functioning, of their schools, and the opening to the collaboration of non Jesuit naturalists, such as the botanists from Oporto, also contributed to the success of Broteria.

KEY WORDS: Broteria. Gonçalo Sampaio. Jesuit botanists. Flora. Portugal.

\section{INTRODUCCIÓN}

El final del siglo XVIII-inicios del XIX, fue un período áureo para el estudio de la flora portuguesa, con la labor de dos personajes excepcionales: Felix de Avellar Brotero (1744-1828) ${ }^{1}$ y Heinrich Friedrich Link ${ }^{2}$. Sin embargo, un siglo después, la botánica portuguesa carecía de modernización. En esta tarea modernizadora participó Gonçalo Sampaio ${ }^{3}$, en colaboración con una red de

1 Cf.: Fernandes, A. (1944), Felix de Avellar Brotero e a sua obra, Boletim da Sociedade Broteriana, série II, XIX (1), pp. LIII-LXXVI; PIRES DE LIMA, A. (1944), Brotero e a sua lição, Boletim da Sociedade Broteriana, série II, XIX (1), pp. XIX-XL; VeIGA, A.B.C. (1945), Félix de Avelar Brotero, Revista da Faculdade de Ciências da Universidade de Coimbra, XIV, pp. 5-14; CABral, J.P. y Folhadela, E. (2006), 3. Botânica. Gonçalo Sampaio. Catálogo da exposição. Ciclo de Exposições "Aventureiros, Naturalistas e Coleccionadores», Porto, Reitoria da Universidade do Porto.

2 Cf.: SACHS, J. vON (1906), History of Botany (1530-1860), Oxford, at the Clarendon Press, edición fac-simile de Kessinger Publishing's Rare Reprints, pp. 267-268; MAGNINGonZe, J. (2004), Histoire de la Botanique, Paris, Delachaux et Niestlé; p. 194; CABRAL y FOLHADELA (2006).

3 Gonçalo Sampaio (1865-1937) fue naturalista del Academia Politécnica de Oporto, director del Herbario y profesor de Botánica de la Facultad de Ciencias de Oporto. Fue especialista en la taxonomía de las plantas vasculares y de los líquenes. Presentó ideas originales acerca de las reglas de la nomenclatura botánica. Estudió y revalorizó las floras clásicas de Portugal de Brotero, Link y van Hoffmansegg. Organizó y distribuyó una exsicata de líquenes - «Lichenes de Portugal»-, constituida por tres centenas de ejemplares. Organizó un herbario rico con millares de ejemplares de todo el país, que aún existe en el Departamento de Biología de la Facultad de Ciencias de Oporto. Escribió una Flora de Portugal, que fue publicada póstumamente en 1946. Cf. publicaciones del autor de esto trabajo: CABRAL, J.P. (2007), A fundação da botânica moderna em Portugal-Júlio Henriques, A. X. Pereira Coutinho e Gonçalo Sampaio, Departamento de Botânica da Universidade de Coimbra, Memórias da Sociedade Broteriana, XXXIII; CABRAL, J.P. (2008), Gonçalo Sampaio y la taxonomia botánica. Conflictos e innovación, Botanica Complutensis, XXXI, pp. 9-19; CABRAL, J.P. (2008), Gonçalo Sampaio e Baltasar Merino, e o estudo da Flora ibérica. Análise de documentación epistolar, Mur- 
botánicos, entre los que destacan los jesuitas de la revista Broteria. Expulsados de Portugal en el siglo XVIII por iniciativa del Marqués de Pombal, los jesuitas regresaron a Portugal a mediados del XIX. A principios del siglo XX fundaron la revista Broteria y empezaron un trabajo notable sobre estudios de la fauna y flora portuguesa.

El presente trabajo intenta analizar el contenido de Broteria, desde su fundación hasta mediados de la década de 1920, y estudiar cual fue su contribución al conocimiento de la historia natural y la formación de colecciones biológicas de Portugal. Pretende también analizar el intercambio epistolar entre los fundadores de Broteria y G. Sampaio ${ }^{4}$, e intenta reconstruir de qué forma, y en qué medida, los jesuitas botánicos y G. Sampaio, contribuyeron a la formación y al desarrollo de la botánica moderna en Portugal.

\section{LA REVISTA BROTERIA (1902-1910): SUS FUNDADORES, CONTENIDO Y FILO- SOFÍA}

La revista Broteria inició su publicación en 1902 bajo el subtítulo «Revista de Sciencias Naturaes do Collegio de S. Fiel». Encontramos en las primeras páginas una fotografía de la estatua de Brotero, que existe en el

guía (Revista Galega de Historia), XV/XVI, pp. 111-136; CABRAL, J.P. (2009), Gonçalo Sampaio y el estudio moderno da la flora ibérica. Análisis de manuscritos epistolares, Boletín de la Real Sociedad Española de Historia Natural (Sección Biológica), CIII, pp. 9-26; CABRAL, J.P. (2009), Gonçalo Sampaio. Professor e botânico notável, Editora da Universidade do Porto, Porto; CABRAL, J.P. (2009), Gonçalo Sampaio. Vida e obra - pensamento e acção, Edição da Câmara Municipal da Póvoa de Lanhoso, Póvoa de Lanhoso.

4 En el Archivo del Departamento de Biología de la Facultad de Ciencias de la Universidad de Oporto (DB-FC-UP) existe un importante conjunto de documentos epistolares inéditos, de destacados botánicos, nombradamente de los jesuitas de la revista Broteria, enviadas a G. Sampaio. El legado epistolar está constituido por cartas y tarjetas postales, que pueden ser examinadas, a pedido. Transcribimos los documentos epistolares de acuerdo con la ortografía original, por eso muchas palabras no tienen tildes. Algunas palabras fueron omitidas porque no son importantes en el contexto de las frases, o porque no eran legibles. Sin embargo, en ningún caso, estas omisiones han puesto en duda el sentido de la frase. Estas omisiones están señaladas por (...). Nuestros comentarios están entre paréntesis rectos. Las transcripciones están entre comillas. Los nombres de los taxones los hemos conservado con la ortografía original (algunos han cambiado de ortografía de acuerdo con las reglas modernas de nomenclatura botánica). Muchos de los documentos epistolares citados en esto trabajo están digitalizados y disponibles en el sitio del Museo Virtual de la Universidad de Oporto: https://museuvirtual.up.pt/. 
Jardín Botánico de la Universidad de Coimbra. En este primer tomo de Broteria, se iniciaba la publicación de una serie de biografías de naturalistas portugueses notables, escritas en latín, por J.S. Tavares. El primero autor era, naturalmente, Brotero. J.S. Tavares justificaba la selección del autor y de la lengua utilizada:

«Neste volume dou principio a uma serie de biographias em latim, em ordem a fazer conhecida no estrangeiro a vida dos naturalistas portugueses. Era justo que começasse por Brotero, cujo nome tomou a nossa Revista» ${ }^{5}$.

Eligiendo una designación asociada al ilustre botánico portugués de los siglos XVIII-XIX, Broteria asumía el papel de revista de Botánica y de las Ciencias Naturales en general. La introducción al primer tomo, se iniciaba con la frase:

«A esperança de podermos concorrer de algum modo para o progresso das sciencias naturaes em Portugal é que nos anima á publicação da presente Revista» ${ }^{6}$.

Broteria pretendía contribuir, con modestia y humildad, engrosando el «ejército» de los naturalistas portugueses:

«Bem sabemos que não passará d'um grãosinho no celleiro immenso dos conhecimentos scientificos; mas primeiramente, quem dá o que tem, não dá pouco; e depois talvez nossos humildes trabalhos tenham a fortuna de incitar outros ingenhos da nossa terra a dedicaram-se a um ramo de saber humano tão interessante e tão vasto» ${ }^{7}$.

El naturalista debe contemplar, respetar y amar su objeto de estudio: la Naturaleza 8 :

5 TAvares, J.S. (1902), Rerum Naturalium in Lusitania Cultores - Felix d'Avellar Brotero, Broteria, I (IX-XIII); p. IX.

6 Duas palabras de Introducção, Broteria, I, pp. V-VII. El texto era firmado por los redactores de la revista.

7 Duas palabras de Introducção, Broteria, I, p. V.

8 Acerca de la pedagogía de las ciencias naturales vista por la Compañía de Jesús, cf.: GorgueS, J.I.C. (1996), Los religiosos en la Sección de Valencia de la Real Sociedad Española de Historia Natural (1913-1936), Madrid, Real Sociedad Española de Historia Natural, pp. 484487; Franco, J.E. (2003a), História da revista Brotéria (1902-2002). En Rico, H. y FrAnCO, J.E. (coords.), Fé, Ciência, Cultura: A Brotéria - 100 anos, Lisboa, Gradiva, pp. 89-139. 
«A idéa de concorrermos, por pouco que seja, para propagar o gosto das sciencias naturaes em nosso patria enche-nos de alegria. A natureza é um livro immenso, que tem ainda muitas folhas por abrir» ${ }^{9}$.

A continuación asumen su actitud cristiana —el amor a Dios pasa por el amor a la Naturaleza-, su Obra:

«Ora em todas ellas [as folhas do livro] se encontra escripto o nome augusto do Creator. E será acaso pequena satisfação ao abril-as mostrar nellas a grandeza de Deus, que tanto se estampa na immensidade dos mundos, como na extrema pequenez de myriades de animaes e plantas, cuja existencia só o microscopio nos revela? (...) Desenvolver as sciencias naturaes é pois, como que dar a mão á intelligencia para a elevar á suprema verdade que é Deus» ${ }^{10}$.

Probablemente debido a la presencia entre los fundadores de la revista, de un experto en microscopia (C. Zimmermann), la anatomía y la histología eran dos temas a abordar en Broteria, después de los tradicionales estudios de flora y fauna:

«Sendo nosso intento nesta Revista attender a tudo quanto possa contribuir para o adiantamento das sciencias naturaes, não nos limitaremos ao estudo systematico da fauna e flora, mas procuraremos, quando couber em nossas forças, tocar outros ramos de maior alcance na historia natural, como são a anatomia e a histologia tanto animaes como vegetaes ${ }^{11}$.

Los estudios de flora y fauna se enfocaban naturalmente en las cercanías del Colegio de S. Fiel, donde estaba localizada la sede de la Dirección de Broteria:

«As nossas explorações teem tido por campo principalmente uma faixa de terreno, que vae dos arredores de Castello Branco até Manteigas e Ceia, numa extensão de 70 kilometros de comprimento sobre 15 a 20 de largura, comprehendendo boa parte da serra da Estrella e toda a da Gardunha, em cujas faldas, ao sul, fica situado este Collegio» ${ }^{12}$.

Sin embargo, la publicación regular de una revista científica, en Portugal, en los comienzos del siglo 20, era tarea osada debido al reducido número de

9 Duas palabras de Introducção, Broteria, I, p. V.

10 Duas palabras de Introducção, Broteria, I, p. V.

11 Duas palabras de Introducção, Broteria, I, p. VI.

12 Duas palabras de Introducção, Broteria, I, p. VI. 
naturalistas y concomitante dificultad en obtener una colaboración regular y, también, a los eternos problemas económicos y financieros asociados a la edición de revistas científicas - publicaciones destinadas a públicos poco numerosos-, no obstante de alguna capacidad financiera. Ya existían otras revistas portuguesas de ciencias naturales, y por tanto, Broteria tenía que disputar su espacio e intentar ganar un grupo de lectores regulares ${ }^{13}$. La publicación de Broteria tenía al problema adicional que sus fundadores eran profesores, y la enseñanza es tarea que exige mucho tiempo y dedicación ${ }^{14}$. Terminan la introducción homenajeando a su patrón, Brotero, y prometiendo seguirle en su trayecto intelectual ${ }^{15}$.

En este primer tomo de Broteria, C. Zimmermann inicia la publicación de un extenso trabajo intitulado «Microscopia vegetal» ${ }^{16}$. En la introducción, su autor escribe sobre la pedagogía de las ciencias naturales. Sus puntos de vista revelan una correcta percepción de cómo se debe enseñar una ciencia experimental como la biología ${ }^{17}$.

Empieza por destacar la importancia de la microscopía en el avance de las Ciencias Naturales:

«Quem acompanhar sequer de longe o actual movimento scientifico, não ignora decerto o maravilhoso auxilio que a microscopia veio prestar ás sciencias nos últimos cincoenta annos ${ }^{18}$. Com os delicados processos de coloração, com a quasi inexcedível perfeição dos microtomos e outros meios de que actualmente dispõe, dilatou extraordinaria e rapidamente o horizonte dos conhecimentos humanos. Digam-no a anatomia animal ou vegetal, a cytologia, a histologia, a physiologia, a

13 Mendes, C.A. (1913), A Broteria no exílio. Broteria Suplemento de Março-Abril de 1913 , p. 5.

14 Duas palabras de Introducção, Broteria, I, p. VI.

15 Duas palabras de Introducção, Broteria, I, p. VII.

16 Zimmermann, C. (1902), Microscopia vegetal, Broteria, I, pp. 49-75. El texto continuaba en Broteria, II, pp. 5-40, IV, pp. 137-159 y V, pp. 229-244.

17 Acerca de la pedagogía moderna de las ciencias naturales en la enseñanza preuniversitaria, cf.: PAN, I. DEL (1949a), Influencia de la Real Sociedad Española de Historia Natural en la divulgación y enseñanza de las Ciencias Naturales, Madrid, Real Sociedad Española de Historia Natural-CSIC, pp. 29-44; PAN, I. DEL (1949b), Bases para un proyecto de Museo Pedagógico-Cultural de Ciencias Naturales en España, Memorias de la Real Sociedad Española de Historia Natural, pp. 261-303.

18 El impacto del microscopio en el conocimiento del mundo vivo remonta el siglo 17, con los trabajos de tres notables botánicos, Malpighi, Grew y van Leeuwenhoek, que son considerados los fundadores de la histología. Cf.: DE WiTT, H.C.D. (1992), Histoire du development de la Biologie, Lausanne, Presses Polytechniques et Universitaires Romandes, p. 286. 
bacteriologia e todos os outros numerosos ramos das sciencias que ao microscopio devem os progressos que estão fazendo» ${ }^{19}$.

La importancia del microscopio era haber dado a conocer un mundo nuevo, antes desconocido, porque no se vía ${ }^{20}$ :

«Revelou-nos ella [microscopia] um novo mundo ou multidão de seres notaveis pela fórma, propriedade e sobretudo pelo numero incalculable, os quaes por sua pequenez se furtavam aos olhos e cuja existencia apenas suspeitaram os homens mais sabios de quantas gerações nos precederam. Presentemente, porém, pela microscopia ficam sob o domínio e ao alcance dos nossos sentidos; estudamse, analysam-se, admira-se-lhes a natureza e importância capital e de tudo isto se auferem vantagens importantissimas para a medicina, hygiene, agricultura, etc. $»^{21}$.

Para C. Zimmermann, la microscopía se podía y debía enseñar y practicar en los Institutos, y no estar restringida a las Universidades. El Colegio de S. Fiel estaba, también en este particular, en la vanguardia de la enseñanza en Portugal 22 .

El fundamento de la pedagogía de las ciencias naturales consistía, para C. Zimmermann, en hacer que los alumnos amasen su objeto de estudio - el mundo vivo y la Naturaleza-. Inculcar este amor era más importante que la propia transmisión de conocimientos, del profesor al alumno. Zimmermann hace imbuir la admiración (el amor) por la Naturaleza, en su misma observación. No tiene duda de que es posible contemplar la Naturaleza y observarla científicamente.

«Os que já ensinaram, sabem perfectamente que o adeantamento de uma aula não depende tanto da maior ou menor sciencia e aptidão do professor, da maior ou menor intelligencia dos discipulos, como do amor que estes mesmos tomam á materia que lhes é ensinada. Se um professor conseguir infundir nos animos juvenis, que o escutam, amor e enthusiasmo para com o que devem estudar, o aliás duro trabalho de ensinar e aprender parece desapparecer por completo. Nada é então difícil, nenhuma lição é demasiadamente grande»» ${ }^{23}$.

19 ZIMMERMANN (1902), p. 49.

20 La descubierta del microscopio también obligaba a entender mejor, con más rigor y exactitud, lo que se observaba (SACHS (1906), pp. 220-222).

21 ZIMMERMANN (1902), p. 49.

22 ZIMMERMANN (1902), pp. 49-50.

23 ZIMMERMANN (1902), p. 53. 
Entonces, la microscopía era ideal para transmitir este amor y entusiasmo:

«Ora nada ha no estudo da anatomia vegetal que tanto excite este amor e interesse no discipulo, como o estudo d'ella por meio do microscopio nos exemplares naturaes. Mostrae aos alumnos algumas preparações no microscopio. Vêem o que nunca viram nem tinham imaginado, e prorompem em admiração e enthusiasmo. Olham, e não se fartam de olhar para os diferentes objectos, fascinados já da elegância, já da variedade, já da quasi geometrica symetria na disposição das partes» ${ }^{24}$.

Naturalmente, Zimmermann, padre jesuita, entendía la observación de la naturaleza usando el microscopio como

«um grande incentivo e poderoso estimulo de piedade e amor de Deus, que tudo fez e ordenou com medida, peso e sabedoria summa» $»^{25}$.

\section{El amor a la Naturaleza era una honra a Dios:}

«Quantas vezes, extasiado e como que arroubado ante as maravilhas que nas minhas preparações o microscopio me patenteava, eu louvei a Deus, tão minucioso, delicado e perfeito em suas obras! $»^{26}$.

C. Zimmermann destaca, correctamente, la importancia primordial de la observación, en las Ciencias Naturales en general y en la microscopía en particular:

«Uma qualidade indispensavel e o primeiro requisito para quem se dedica ás sciencias naturaes é incontestavelmente o espirito de observação. Sem elle o naturalista assemelhar-se-hia a um viajante melancólico e apático que, atravesando verdejantes prados e campos cheios de fructos, não reparasse nas variegadas boninas e flores de que estão matizados, cerrasse os ouvidos ao alegre chilrear das avezinhas e não se deixasse impressionar dos encantos da paizagem que o rodeia. Ora espirito de observação, como este, é o que requer em alto grau a microscopia; mas não o requer só: desenvolve-o e aperfeiçoa-o extraordinariamente» ${ }^{27}$.

Y la función del profesor, era aclarar las dudas suscitadas por la observación al microscopio:

24 ZIMMERMANN (1902), p. 53.

25 ZIMMERMANN (1902), p. 56.

26 ZIMMERMANN (1902), p. 56.

27 ZIMMERMANN (1902), pp. 54-55. 
«Depois vem uma multidão de perguntas, a que é mister satisfazer. Tudos querem saber, e de tudo dar razão. O primeiro impulso está dado áquelles espíritos juvenis. Aprenderam n'uma hora o que não aprenderiam em muitos dias sem o microscopio. Vereis então os noveis anatomistas com ideias claras e precisas das coisas, em logar das ideias vagas, ligeiras e muitas vezes incorrectas e falsas que adquiriram no estudo dos livros, embora adornados de estampas, estudo que ordinariamente se faz aborrecer por fatigar a memoria. O que se vê grava mais profundamente que o que se leu ou ouviu» ${ }^{28}$.

Partir siempre de la observación para la elaboración de interpretaciones explicativas y regresar nuevamente a la observación para confirmar las hipótesis presentadas; esto era, para C. Zimmermann, el método correcto de progresar en las investigaciones biológicas, en general, y en las observaciones microscópicas en particular ${ }^{29}$.

¿Quiénes eran los fundadores de la revista Broteria $^{30}$ ? P. ${ }^{\mathrm{e}}$ Joaquim da Silva Tavares $^{31}$, P. $^{\mathrm{e}}$ Carlos Zimmerman ${ }^{32}$ y P. ${ }^{\mathrm{e}}$ Candido d'Azevedo Mendes ${ }^{33}$, profesores del Colegio d S. Fiel. Estos tres profesores dirigían la revista y tenían como colaboradores a P. ${ }^{\mathrm{e}}$ Alphonse Luisier ${ }^{34}$, a P. ${ }^{\mathrm{e}}$ Antonio da Costa e Oliveira

28 ZIMMERMANN (1902), pp. 53-54.

29 ZIMMERMANN (1902), pp. 53-54.

30 Biografías de los fundadores y directores de la revista se encuentran en: FrANCO, J.E. (2003b), Os directores da Brotéria. En Rico, H. y FrAnCO, J.E. (coords.), Fé, Ciência, Cultura: A Brotéria - 100 anos, Lisboa, Gradiva, pp. 143-190. Los elementos biográficos que se presentan seguidamente se focalizan en el período hasta 1910, y fueron obtenidos de FRANCO, J.E. (2003b) y de la Grande Enciclopédia Portuguesa e Brasileira. La continuación de las biografías después de 1910 se encuentran en el capítulo siguiente.

31 J.S. Tavares (1866-1931) nació en Cardigos, Vila de Rei, Portugal. Terminó su formación teológica en Viena (Austria) en 1899. Enseñó portugués, latín, griego, física, química, ciencias naturales e historia natural en los Colegios de Setúbal, Campolide y de S. Fiel. Fue Director del Herbario y del Museo de Historia Natural del Colegio de S. Fiel.

32 C. Zimmermann (1871-1950) nació en Alemania. En 1890 viene a Portugal. Terminó sus estudios teológicos superiores en Inglaterra e Irlanda, en 1906. Enseñó latín, música, alemán, física, química, historia, geografía y dibujo en el Colegio de S. Fiel.

33 C.A. Mendes (1874-1943) nació en Soutos, Torres Novas, Portugal. Realizó sus estudios teológicos superiores en Roma en 1902-1907. Enseñó ciencias físicas, químicas y naturales en el Colegio de S. Fiel.

34 A. Luisier (1872-1957) nació en Fregnoley, Valais, Suiza. Terminó sus estudios teológicos en Innsbruch (Austria), en 1906. Enseñó ciencias naturales en el Colegio de Campolide. 
Pinto, a P. ${ }^{\mathrm{e}}$ Camillo Torrend ${ }^{35}$, a P. ${ }^{\mathrm{e}}$ Longinos Navás ${ }^{36}$ y a $\mathrm{P} .{ }^{\mathrm{e}}$ Manuel Maria Rebimbas Tavares ${ }^{37}$.

¿Cuáles eran sus especialidades científicas y sus publicaciones en Broteria (1902-1907)? J.S. Tavares, entomólogo temprano, se especializó en el estudio de las zoocecidias, las deformaciones inducidas por insectos en plantas. Había publicado, en 1900, un primer catálogo de las zoocecídias portuguesas (continente) $^{38}$. En 1902 y 1903, publica adendas a este catálogo ${ }^{39}$. En 1905, publica una sinopsis de las zoocecídias portuguesas ${ }^{40}$ y en 1903 inicia la publicación de la serie «Revista Annual de Cecidologia», donde pretendía dar a conocer las novedades en esta área de estudio ${ }^{41}$. Estudia también las cecídias de la Isla de Madeira ${ }^{42}$.

C. Zimmermann se especializa en microscopía, principalmente en el estudio de las diatomeas. En 1906, empieza la publicación de un catálogo de las especies portuguesas ${ }^{43}$.

C.A. Mendes manifiesta temprano una predilección especial por el estudio de los insectos lepidópteros. Recoge ejemplares en los contornos del Colegio de S. Fiel y publica catálogos de esta región ${ }^{44}$. En 1904 inicia la publicación de la serie «Revista bienal de lepidopterologia», donde pretendía dar a conocer las novedades científicas en esta área de estudio ${ }^{45}$.

A. Luisier inicia en 1904 la publicación de la serie «Revista de Bryologia», donde pretendía dar a conocer los últimos desarrollos en esta especiali$\mathrm{dad}^{46}$.

35 C. Torrend nació en Saint-Privat d'Allier, Velay, Francia, en 1875. Viene a Portugal, en 1894. Desde 1900 hasta 1902, estudia filosofía en el Colegio de Setúbal. Enseñó francés e historia natural, en el Colegio de S. Fiel. Terminó sus estudios teológicos en Irlanda, en 1907.

36 L. Navás (1858-1941) nació en Cabacés, Tarragona. Realizó sus estudios teológicos en Barcelona. Enseñó Historia Natural en el Colegio de S. Salvador, en Zaragoza, y en Colegio de Madrid. En 1902, fundó la Sociedad Aragonesa de Ciencias Naturales.

37 Rebimbas Tavares (1873-1944) nació en Pardelhas, Murtosa, Portugal. Terminó sus estudios teológicos en Bélgica. Enseñó en el Colegio de S. Fiel.

38 TAVARES, J.S. (1900), Catálogo de las zoocecídias portuguesas, Annaes de Sciencias Naturaes, VII, pp. 17-108.

39 Broteria, I, pp. 3-48 y II, pp. 160-179.

40 Broteria, V, pp. v-123.

41 Broteria, II, pp. 81-86 y IV, pp. 227-230.

42 Broteria, II, pp. 179-186 y IV, pp. 221-227.

43 Broteria, V, pp. 245-251.

44 Broteria, I, pp. 151-171; II, pp. 41-80; III, pp. 223-254 y IV, pp. 166-175.

45 Broteria, III, pp. 264-276 y V, pp. 252-257.

46 Broteria, III, pp. 254-263 y V, pp. 115-124. 
C. Torrend, micólogo temprano, estudia los hongos (macromicetos) de las cercanías del Colegio de Setúbal, publicando dos trabajos ${ }^{47}$. Recoge material en el contorno del Colegio de S. Fiel ${ }^{48}$. Estudia los hongos (macromicetos) de Mozambique recogidos por su hermano, P. ${ }^{\mathrm{e}}$ Julio Torrend, y por P. ${ }^{\mathrm{e}}$ Luiz Gonzaga Dialer ${ }^{49}$.

L. Navás, entomologista, estudia, en particular, los neurópteros de la Península Ibérica ${ }^{50}$.

Rebimbas Tavares publica regularmente trabajos acerca de temas de física y química, como la radioactividad.

La sede de Broteria estaba en el Colegio de São Fiel, en Louriçal do Campo, cerca de Castelo Branco, en el centro-interior del país.

Pasados algunos años, en 1907, la revista se divide en tres series, una de divulgación científica, otra de botánica y otra de zoología. La serie de divulgación científica tiene varias secciones: 1 . Historia de las Ciencias Naturales en Portugal. 2. Fisiología animal. 3. Fisiología vegetal. 4. Técnica microscópica. 5. Microbiología. 6. Medicina. 7. Física. 8. Química. 9. Animales útiles y nocivos. 10. Arboricultura. 11. Variedades. 12. Bibliografía.

Los fundadores se mantienen en la redacción de Broteria, pero el equipo de redactores se amplia. En la introducción al tomo sexto, con fecha de ese año, se hace un balance positivo de los primeros cinco años de actividad ${ }^{51}$.

Con tres series, Broteria amplía considerablemente su volumen de publicación. Sus estudios, ahora, «extravasan» el territorio continental para enfocar a otras regiones, como las Islas Atlánticas portuguesas, el Brasil, África e incluso el Timor portugués, en el Asia distante. Aunque los jesuitas fundadores decidan viajar, más antes, establecen una red de contactos (naturalistas que son también colectores y sus corresponsales).

¿Qué trabajos son publicados en Broteria por sus fundadores, desde 1907 hasta 1910 ?

J.S. Tavares publica trabajos de divulgación acerca de temas variados de botánica $^{52}$ y zoología. Publica un apéndice al catálogo de zoocecidias portu-

47 Broteria, I, pp. 94-150; II, pp. 123-148 y IV, pp. 207-211.

48 Nombradamente hongos mixomicetos, que serán estudiados durante la estancia en Dublín, donde terminará su formación teológica.

49 Broteria, IV, pp. 212-221.

50 Broteria, II, pp. 107-113 y V, pp. 145-184.

51 Broteria, serie de Vulgarização Scientifica, VI.

52 J.S. Tavares publica una serie amplia de interesantes trabajos acerca de los árboles notables de la región interior centro del país. 
guesas, editado en $1905^{53}$ y un estudio preliminar de las zoocecidias de Zambia $^{54}$ y de Brasil ${ }^{55}$.

C. Zimmermann prosigue sus investigaciones acerca de las diatomeas. Publica un trabajo de divulgación científica ${ }^{56}$, continúa el catálogo de las diatomeas portuguesas (continente) ${ }^{57}$ y empieza la publicación del catálogo de las diatomeas de las Islas Madeira y Porto Santo ${ }^{58}$.

C.A. Mendes continúa el estudio de los lepidópteros de Portugal ${ }^{59}$ y publica un trabajo acerca de los lepidópteros de los contornos de Roma ${ }^{60}$, recogidos cuando estuvo estudiando en la capital italiana.

A. Luisier empieza la publicación de una exsicata de musgos portugueses: la «Bryotheca Lusitanica» ${ }^{61}$.

A.C. Oliveira Pinto continúa la publicación de trabajos de divulgación acerca de la física y química.

C. Torrend prosigue los estudios micológicos en Irlanda, donde estaba terminando su formación teológica. Publica un trabajo de divulgación científica ${ }^{62}$, empieza la publicación de una flora de los mixomicetos portugueses ${ }^{63}$ y de los hongos (macromicetos) de Timor, con material recogido por el P. ${ }^{\text {e }}$ Emmanuel Ferreira ${ }^{64}$. Publica una especie nueva de Amanita (A. lusitanica Torrend), con diagnosis en latín ${ }^{65}$.

L. Navás estudia entomología y liquenología. Continúa la publicación del catálogo de los neurópteros peninsulares ${ }^{66}$. Inicia la publicación del

53 Broteria, serie Zoologica, VI, pp. 109-134.

54 Broteria, serie Zoologica, VII, pp. 133-171.

55 Broteria, serie Zoologica, VIII, pp. 5-36.

56 Broteria, serie de Vulgarização Scientifica, IX, pp. 42-51.

57 Broteria, serie Botanica, VIII, pp. 89-103 y IX, pp. 95-102.

58 Broteria, serie Botanica, VIII, pp. 114-127 y IX, pp. 103-120.

59 Broteria, serie Zoologica, VIII, pp. 67-73 y IX, pp. 60-134.

60 Broteria, serie Zoologica, IX, pp. 135-162.

${ }_{61}$ La exsicata estaba organizada en conjuntos de 25 especies. El primero estaba fechado el abril de 1910. En la serie botánica (IX, pp. 67-68) donde se publicaban las descripciones de las especies. La exsicata era distribuida, en Alemania, por Thedor Oswald Weigel, Leipzig.

62 Broteria, serie de Vulgarização Scientifica, IX, pp. 95-106.

63 Broteria, serie Botanica, VI, pp. 5-64; VII, pp. 5-177 y IX, pp. 45-52. En 1909, publica, como suplemento a su flora, una traducción de una obra de A. y G. Lister (Broteria, serie Botanica, VIII, pp. 5-30).

64 Broteria, serie Botanica, IX, pp. 83-91.

65 Broteria, serie Botanica, IX, pp. 92-94.

66 Broteria, serie Zoologica, VI, pp. 43-100 y VII, pp. 5-131. 
estudio de los líquenes de las Islas Azores, con ejemplares recogidos por Bruno Tavares Carreiro, médico en Ponta Delgada ${ }^{67}$.

Rebimbas Tavares publica varios trabajos de divulgación, principalmente acerca de la fisiología humana.

En la serie botánica son publicados varios trabajos acerca de la flora de la Isla Madeira: A. Luisier estudia los musgos ${ }^{68}$, C. Torrend los hongos (macromicetos) ${ }^{69}$ y L. Navás los líquenes ${ }^{70}$.

\section{LOS JESUITAS DE BROTERIA Y GONÇALO SAMPAIO (1901-1910)}

A pesar de que G. Sampaio publica su primer trabajo en Broteria en $1916^{71}$, los contactos entre el y los fundadores de la revista se remontan a muchos años antes, iniciándose en la década de 1900. G. Sampaio tuvo un largo y rico intercambio epistolar y botánico con A. Luisier y J.S. Tavares, que se prolongó durante más de dos décadas, desde el inicio de 1900 hasta después de 1920. El análisis de estos documentos permite una reconstitución histórica minuciosa del intercambio científico entre los botánicos de Broteria y G. Sampaio, y evaluar la real contribución de estos naturalistas al avance de la botánica portuguesa.

La primera carta de A. Luisier a G. Sampaio está fechada de 1901. Luisier vivía en el Colegio de Setúbal. Había recibido un trabajo y pliegos de herbario de Sampaio y le escribió agradecido:

«Recebi as estimadas cartas de V. Ex. a que muito agradeço. Não menos penhorado fiquei com o offerecimento do interessantissimo artigo sobre a flora do Torrão ${ }^{72}$. A flora d'aquella região parece bastante semelhante á dos arredores de Setubal; comtudo va-

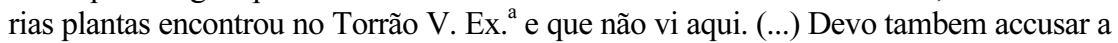

67 Broteria, serie Botanica, VIII, pp. 46-52.

68 A. Luiseri había publicado dos trabajos en el Bulletin de la Société Portuguaise de Sciences Naturelles. Continuaba en Broteria, serie Botanica, VIII, pp. 31-45 y IX, pp. 54-66.

69 Broteria, serie Botanica, VIII, pp. 128-144.

70 Broteria, serie Botanica, IX, pp. 69-82.

71 SAmpaio, G. (1916a), Líquenes novos para a flora portuguesa (1. a série), Broteria, serie Botanica, XIV, pp. 65-84.

72 A. Luisier se refería al trabajo de SAMPAIO, G. (1901a), Um passeio botanico ao Torrão, Boletim da Sociedade Broteriana, XVIII, pp. 47-79. 


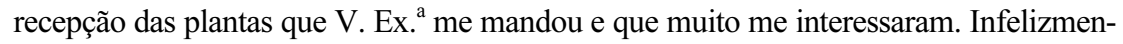
te tive ainda apenas o tempo de examinar algumas. (...) $\rangle^{73}$.

Del Colegio de Setúbal, Luisier parte hacia Innsbruck, donde realizó sus estudios teológicos. Inicialmente, estudia las plantas vasculares, pero rápidamente su interés se centra en el estudio de los musgos y hepáticas (briofitas). Luisier pedía ayuda a Sampaio sobre algunos musgos portugueses:

«Dirigo me mais uma vez a V. Ex. ${ }^{a}$ depois de um longo silencio, para lhe pedir mais um favor. O Sr. G. Roth que está publicando a sua bella obra (...) acaba de me escrever para me pedir que eu lhe procurasse, sendo possível, as seguintes 4 especies de musgos hespanhoes e portuguezes que elle não poude ainda desenhar para a sua obra, por falta de exemplares originais: (...) Se pois fosse possivel arranjar um pequeno numero de bons exemplares d'alguma d'estas especies, ficaria eu muito agradecido a V. Ex. ${ }^{a}$ por este novo favor. Sendo preciso, estes exemplares lhe serão cuidadosamente reenviados. (...) Eu aqui continuo a dar os meus momentos livres ao estudo da flora alpina destes arredores e em especial dos musgos» ${ }^{74}$.

A. Luisier terminaba esta carta elogiando el estudio que G. Sampaio estaba realizando sobre la flora portuguesa:

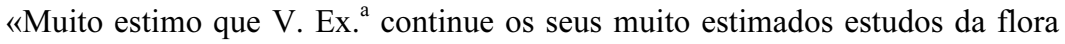
portugueza. Soube pelo pequeno artigo bibliographico publicado no ultimo $\mathrm{n} .^{\circ} \mathrm{da}$ Broteria que V. Ex. ${ }^{a}$ tinha publicado o Catalogo da Cryptogamia do herbario da Academia Polytechnica ${ }^{75}$. Muito estimei esta noticia. Tudo o que diz respeito á Flora portugueza, que espero tornar a estudar mais tarde, é para mim de muito interesse $»^{76}$.

De Austria, Luisier regresa a Portugal para enseñar en el Colegio de Campolide en Lisboa. Siendo su interés el estudio de los briofitos, le pide a Sampaio ejemplares de estas plantas. Le escribía agradeciendole el envío de musgos:

73 Carta de A. Luisier a G. Sampaio, fechada el 30 de agosto de 1901, enviada del Colegio de Setúbal (DB-FC-UP).

74 Tarjeta postal de A. Luisier a G. Sampaio, fechada el 14 de marzo de 1904, enviada del Colegio de Innsbruch (DB-FC-UP).

75 A. Luisier se refería al trabajo de SAMPAIO, G. (1902), Herbario Portuguez da Academia Polytechnica do Porto. I. Cryptogamiae, Annuario da Academia Polytechnica, XXV, pp. 81-171.

76 SAMPAIO (1902). 
«Recebi da Livraria Lopes um caixote com tres pastas de musgos. Agradeço cordealmente tão grande favor. Restituilas hei antes do mes d'outubro. Espero encontrar na colleção bom material para os meus estudos bryologicos» ${ }^{77}$.

Efectivamente, había ejemplares muy interesantes. Luisier escribió a Sampaio en septiembre, pidiéndole para recoger, en sus herborizaciones, más musgos. A cambio, le enviaría sus duplicados:

«Estive estes dias a examinar os musgos da Academia Polyt. [Polytechnica] que V. Ex. ${ }^{a}$ me mandou. No meio de um Dicrarum seojanicum colhido por V. Ex. ${ }^{a}$ em Ponte de Lima, no logar chamado Formigoso, encontrei um Campylopus que me parece ser o $C$. flexuosus. Infelizmente não tem esporófitos. Se for essa especie, é novo para Portugal. Daqui a alguns dias irei para o Collegio do Barro, Torres Vedras, onde tenciono demorar-me até ao mez de Agosto de 1908, mas em occupações que não darão muito logar a estudos botanicos. Peço instantemente a V. Ex. ${ }^{\text {a } q u e ~ m e ~ g u a r d e ~ a l g u n s ~ e x e m p l a r e s ~ d o s ~ m u s g o s ~ q u e ~ e n c o n t r a . ~ B r e v e m e n t e ~ r e-~}$ metterei para o Porto os musgos que V. Ex. ${ }^{\mathrm{a}}$ me mandou com mais alguns» ${ }^{78}$.

Pero A. Luisier no logra estudiar todo el material que G. Sampaio le había enviado antes de partir al Colegio de Torres Vedras y le escribe dos días después, devolviéndole los pliegos, unos identificados y otros no, y pidiéndole para recoger un musgo particular:

«No fim da semana irei a Torres Vedras onde me demorarei varios mezes; por isso não me atrevo a guardar os musgos da Acad. [Academia] Polyt. [Polytechnica]

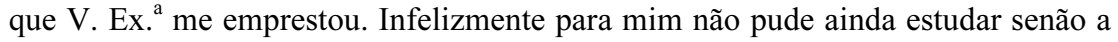

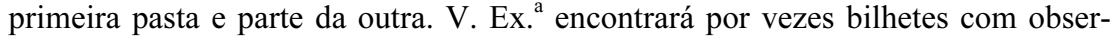
vações minhas. Veja lá se pode encontrar no muro de uma regueira ahi em Rendufinho uma Webera Tozzeri que V. Ex. ${ }^{a}$ alli colheu em 1897 e que me parece ser uma forma interessante» ${ }^{79}$.

Algunos años después, en 1910, ya de regreso en el Colegio de Campolide, Luisier escribía a Sampaio claramente demostrando su continuada pasión por los briofitos:

77 Tarjeta postal de A. Luisier a G. Sampaio, fechada el 12 de junio de 1907, enviada del Colégio de Campolide (DB-FC-UP).

78 Tarjeta postal de A. Luisier a G. Sampaio, fechada el 20 de septiembre de 1907 (DBFC-UP).

79 Tarjeta postal de A. Luisier a G. Sampaio, fechada el 22 de septiembre de 1907 (DBFC-UP). 
«Estou actualmente rodeado de musgos. É preciso aproveitar as ferias para adiantar o trabalho de classificação» ${ }^{80}$.

Naturalmente, J.S. Tavares pretendía mejorar sus colecciones biológicas y, por eso, en la mayoría de las cartas que escribió a G. Sampaio, le pidió ejemplares de cecidias. Sampaio herborizaba con frecuencia, recogía muchas plantas para preparar pliegos de herbario. Naturalmente le era tan fácil recoger musgos para Luisier como cecidias para Tavares. En 1902, Tavares le escribe pidiendole ejemplares de cecidias $^{81}$. En agosto de 1904, Tavares se encontraba en la sierra del Gerês, en el norte de Portugal, recogiendo ejemplares para su colección y escribió a Sampaio, invitándolo a que lo acompañara en sus excursiones, pidiéndole nuevamente ejemplares de cecidias y ofreciéndose para recoger plantas en la sierra ${ }^{82}$. Sampaio se las debió haber enviado, porque Tavares le escribe una carta en diciembre, agradeciéndole y devolviendo los ejemplares de cecidias prestados ${ }^{83}$.

A cambio, Luisier y Tavares enviaban a G. Sampaio pliegos para el Herbario de la Academia Politécnica. La flora portuguesa continental es rica y diversa debido a la influencia simultánea y convergente, en este país, del Atlántico y del Mediterráneo. En esta época, la flora portuguesa, especialmente la del interior y sur, era mal conocida. G. Sampaio vivía en Oporto, en el norte del país. Los transportes no eran fáciles y, por tanto, pretendía recibir plantas de regiones del país que no conocía bien. A. Luisier había vivido en Setúbal y ahora vivía en Lisboa; J.S. Tavares vivía en el Colegio de S. Fiel. Eran, pues, los indicados para recoger plantas de regiones poco familiares a G. Sampaio. Éste no estudiaba la flora portuguesa siguiendo el orden natural de las familias, sino que prefería concentrarse en el estudio de especies interesantes, confusas o mal conocidas de la flora portuguesa. Algunas de estas especies habían sido descritas en las floras de Brotero o de Link y Hoffmansegg, pero habían sido mal interpretadas u olvidadas por otros botánicos ${ }^{84}$.

80 Tarjeta postal de A. Luisier a G. Sampaio, con fecha el 4 de enero de 1910, enviada del Colegio de Campolide (DB-FC-UP).

81 Carta de J.S. Tavares a G. Sampaio, fechada el 10 de enero de 1902, enviada del Colegio de S. Fiel (DB-FC-UP).

82 Carta de J.S. Tavares a G. Sampaio, fechada el 9 de Agosto de 1904, enviada del «Hotel Anselmo, Gerez» (DB-FC-UP).

83 Carta de J. S. Tavares a G. Sampaio, con fecha el 23 de diciembre de 1904, enviada del Colegio de S. Fiel (DB-FC-UP).

${ }^{84}$ Una de las más significativas contribuciones de G. Sampaio al estudio de la flora portuguesa fue efectivamente la revalorización de las floras de Brotero («Flora lusitanica» y «Phytographia Lusitaniae selectior») y de Link y Hoffmansegg («Flore Portuguaise»). 
Así, los envíos de plantas de Luisier y Tavares a Sampaio se centran, frecuentemente, en determinadas especies que estaban siendo revisadas éste. En una carta de 1902, Tavares escribe a Sampaio:

«Creio que o Sr. Zimmermann lhe deve ter já escripto p. ${ }^{a}$ [para] agradecer os lichenes. Quanto á Menthas diz elle que não há difficuldade nenhuma» ${ }^{85}$.

De hecho, G. Sampaio publicó un trabajo sobre el género Mentha fechado el enero de $1902^{86}$. ¿Desearía G. Sampaio consultar las menthas del Herbario de S. Fiel para aclarar algunas dudas o confirmar algunas hipótesis?

En 1904, Tavares se refería a un anterior envío de Rubus e invitaba Sampaio a ir al Colegio de S. Fiel a estudiar los pliegos de su herbario:

«Peço me queira desculpar o ter-lhe enviado pela segundo vez o Rubus hybrido. Olhe que não me lembrava que lh'o tinha mandado ha dois annos, de outra sorte o não faria. Muito prazer nos dará vindo aqui estudar os Rubus. Espero que não serão só as especies d'esse gen. [género] que lhe hão-de interessar aqui» ${ }^{87}$.

Efectivamente, G. Sampaio estudiaba con ahínco, desde hacía algunos años, el género Rubus. En el primer trabajo de la serie «Plantas novas para a Flora de Portugal» ${ }^{88}$, había discutido algunos Rubus. En el primer y segundo trabajo de la segunda serie «Plantas novas» ${ }^{89}$, continuaba esta discusión. Sin embargo, la mayoría de las novedades taxonómicas son publicadas en dos trabajos de la segunda serie de «Plantas novas» publicados en $1903^{90}$, y en

85 Carta de J.S. Tavares a G. Sampaio, fechada el 10 de enero de 1902, enviada del Colegio de S. Fiel (DB-FC-UP).

86 Sampaio, G. (1901b), Nota sobre as especies do genero Mentha dos arredores do Porto, Boletim da Sociedade Broteriana, XVIII, pp. 126-136.

87 Carta de J.S. Tavares a G. Sampaio, fechada el 15 de junio de 1905, enviada del Colegio de S. Fiel (DB-FC-UP).

88 SAmpaio, G. (1899a), Plantas novas para a Flora de Portugal. I, Annaes de Sciencias Naturaes, VI, pp. 67-78.

89 SAmpaiO, G. (1899b), Plantas novas para a Flora de Portugal. II, Annaes de Sciencias Naturaes, VI, pp. 141-151; SAMPAIO, G. (1900), Plantas novas para a Flora de Portugal. III, Annaes de Sciencias Naturaes, VII, pp. 7-14.

90 Sampaio, G. (1903a), Plantas novas para a Flora de Portugal (2. ${ }^{a}$ série). I, Annaes de Sciencias Naturaes, VIII, pp. 5-16; SAMPAIO, G. (1903b), Plantas novas para a Flora de Portugal (2. a série). II, Annaes de Sciencias Naturaes, VIII, pp. 115-122. 
tres trabajos que publica en $1904^{91}$, donde describe varios taxones nuevos para la ciencia y varias nuevas combinaciones de Rubus. Finalmente, en diciembre de 1904, publicaría una importante monografía de los Rubus portugueses $^{92}$. Para hacerla, estudió muchos ejemplares de diversos herbarios portugueses y extranjeros. Por tanto, los del Colegio de S. Fiel también fueron estudiados, como se deduce por esta carta.

En diciembre de 1905, G. Sampaio publicaba uno de sus trabajos de mayor aliento e importancia: las «Notas criticas» $\rangle^{93}$. Se trata de una discusión pormenorizada de 113 especies y variedades de la flora portuguesa. Uno de los taxones discutidos es una planta que había sido descubierta por A. Luisier en las cercanías del Colegio de Setúbal, y que G. Sampaio había propuesto como una especie nueva para la ciencia, llamándola con un nombre sugestivo: Teucrium Luisieri Samp ${ }^{94}$. En este trabajo, proponía considerar esta especie como una variedad de otra ya existente - Teucrium Haenseleri Boiss. var. Luisieri Samp. Otra de las especies discutidas es la Nepeta multibracteata que había sido descrita, tanto por Brotero en su «Phytographia lusitanica», como por Desfontaines en la «Flora Atlantica». G. Sampaio tenía dudas de si Brotero y Desfontaines se estaban refiriendo a la misma planta y cuál de las descripciones tenía prioridad. Rouy había descrito otra Nepeta, la N. lusitanica. ¿Seria efectivamente una especie autónoma o una variedad de otra Nepeta? Para aclarar estos problemas, Sampaio examina los ejemplares de los herbarios portugueses y le pide a Tavares que le envíe los pliegos existentes en el Herbario del Colegio de S. Fiel. Tavares no solamente accede al pedido de Sampaio, sino que intentará recoger más plantas de esto género en las cercanías del Colegio. Aprovecha la oportunidad para pedirle a Sampaio que revisase

91 Sampaio, G. (1904a), Rubus Henriquesii, Samp., A Revista, I (4), pp. 57-59; SAMPAIO, G. (1904b), Alguns Rubus novos para a flora portuguesa. I, A Revista, II (3), pp. 41-44; SAMPAIO, G. (1904c), Alguns Rubus novos para a flora portuguesa. II, A Revista, II (4), pp. 54-57.

92 Sampaio, G. (1904d), Rubus portugueses. Contribuições para o seu estudo, Porto, Typ. de A.F. Vasconcellos.

93 Este trabajo y la monografía acerca de los Rubus portugueses culminan de forma excepcional, 10 años de investigaciones botánicas. El rigor y madurez científica de estos dos trabajos indican que G. Sampaio había evolucionado rápidamente en la taxonomía vegetal y en el conocimiento acerca de la flora portuguesa. La rigurosidad de estas «Notas criticas» se convertirá en el estilo típico de G. Sampaio en sus trabajos futuros, publicados principalmente en el período de 1924-1937.

94 SAMPAiO (1900). 
todos los pliegos enviados de la familia de las Lamiaceae (Labiadas) e insiste en que vaya al Colegio ${ }^{95}$.

En 1909, G. Sampaio inicia la publicación, en fascículos, que se prolongará durante algunos años, de dos de sus obras principales, el «Manual da Flora Portugueza» ${ }^{96}$ y el $«$ Prodromo da flora portuguesa» ${ }^{97}$, que pretendían constituir una nueva Flora de Portugal ${ }^{98}$. Probablemente para aclarar algunas dudas relativas a la taxonomía del género Ulex, Sampaio pidió prestados a Luisier los pliegos de esto género conservados en los herbarios de los colegios jesuitas. Luisier le contesta lamentándose de las deficientes condiciones del Herbario del Colegio de Setúbal y de no poder enviarle buenos pliegos de Ulex ${ }^{99}$.

Los pliegos enviados no estaban en buenas condiciones y Sampaio pidió a Luisier otros, especialmente de Sintra, una sierra cercana a Lisboa, mas con condiciones edáficas y climáticas particulares y con una flora singular, distinta de la flora de las cercanías. Luisier accede el pedido de G. Sampaio, con la evidente satisfacción del naturalista que recogió en la naturaleza lo que procuraba, y relatando sus observaciones personales acerca del difícil género Ulex, con clara intención de ayudar a Sampaio, le escribió pocos días después ${ }^{100}$. Agradecido con las observaciones de Luisier, Sampaio le envía un borrador de la taxonomía de los Ulex. Luisier le contestó elogiando su trabajo y estimulándolo a continuar la publicación del «Manual»101.

95 Carta de J.S. Tavares a G. Sampaio, fechada el 16 de junio de 1905 (post scriptum fechado el 17), enviada del Colégio de S. Fiel (DB-FC-UP).

96 Sampaio, G. (1909-1914), Manual da Flora Portugueza, Porto, Tipografia Occidental. [Obra publicada en fascículos: páginas (ano mes): 1-16 (1909 05), 17-32 (1909 08), 33-48 (1909 12), 49-176 (1910 05), 177-208 (1910), 209-320 (1911 12), 321-336 (1912 01), 337-353 (1912 11), 354-368 (1912 12), 369-384 (1913 11), 385-400 (1914 11), 401-416 (1914 12)].

97 SAmpaio, G. (1909a), Prodromo da flora portuguesa. (I), Annaes da Academia Polytechnica do Porto, IV (1), pp. 36-64, [táxones 1-195]; SAMPAIO, G. (1909b), Prodromo da flora portuguesa. (II), Annaes da Academia Polytechnica do Porto, IV (2), pp. 116-128, [táxones 196-270]; SAMPAIO, G. (1910), Prodromo da flora portuguesa. (III), Annaes da Academia Polytechnica do Porto, V (1), pp. 44-64, [táxones 271-409]; SAMPAIO, G. (1911), Prodromo da flora portuguesa. (IV), Annaes da Academia Polytechnica do Porto, VI (1), pp. 39-57, [táxones 410-557].

98 La Flora de Portugal de G. Sampaio será publicada después de su muerte por sus discípulos en 1946: SAMPAIO, G. (1946), Flora portuguesa, A. Pires de Lima (dir.), Porto, Imprenta Portuguesa, 792 pp. (obra póstuma).

99 Tarjeta postal de A. Luisier a G. Sampaio, con fecha el 7 de julio de 1910, enviada del Colegio de Campolide (DB-FC-UP).

100 Carta de A. Luisier a G. Sampaio, con fecha el 19 de julio de 1910 (DB-FC-UP).

101 Tarjeta postal de A. Luisier a G. Sampaio, fechada el 1 de septiembre de 1910, enviada del Colegio de S. Fiel (DB-FC-UP). 


\section{LOS HERBARIOS DE LOS COLEGIOS JESUITAS}

Los Colegios de S. Fiel, Campolide y Setúbal, «construyen» poco a poco sus herbarios, con las herborizaciones de sus profesores y naturalistas y el intercambio con otros botánicos y Herbarios, como los de la Academia Politécnica de Oporto, dirigido por Sampaio, como hemos visto en el capítulo anterior.

El Herbario del Colegio de S. Fiel se convertirá en uno de los mejores de Portugal. Las colecciones empiezan con C. Zimmermann en 1897102; en 1903 J.S. Tavares asume la dirección hasta 1907, año en que regresa Zimmermann ${ }^{103}$. En Broteria se describían las colecciones de historia natural:

«Possue tambem um herbario, rico de cryptogamicas inferiores - muscineas, algas, fungos e lichens; collecções incompletas de vertebrados portugueses, collecções importantes de lepidópteros da Europa e Africa; orthopteros da Peninsula Iberica, Zoocecidias do globo, e varias outras em começo- nevropteros, coleopteros, dipteros, hymenopteros, etc. Uma boa parte d'estas collecções servem grandemente ao estudo da fauna e flora d'esta região de S. Fiel» ${ }^{104}$.

Las colecciones del Colegio de Campolide están descritas por A.C. Oliveira Pinto en dos trabajos que publica en la revista de los colegios jesuitas portugueses «O Nosso Colégio, 1908-1910»105.

Las mejores colecciones eran de hongos y musgos:

«uma das mais completas collecções de fungos de Portugal; e, pelo que diz respeito á ordem dos myxomycetes é seguramente, depois da do British Museum, a mais completa da Europa (...) das 283 especies conhecidas contem ella 199 especies; e entre estas algumas muito raras e novas para a sciencia [só faltando] quasi exclusivamente especies criticas ou duvidosas ou algumas raríssimas» ${ }^{106}$.

El estudio de los mixomicetos era realizado por C. Torrend. Las colecciones de musgos tenían ejemplares de Isla Madeira y del continente portugués, y eran ampliadas por la compra de:

102 Tavares, J.S. (1924), O Herbário do Collégio de S. Fiel, Broteria, serie Botanica, XXI, pp. 82-87.

103 TAVARES (1924).

104 Broteria, serie de Vulgarização Scientifica, VI, p. 11.

105 Oliveira Pinto (1910a), O Instituto de Sciencias Naturaes do Collegio de Campolide, En O Nosso Colegio, 1908-1910, Lisboa, ed. del Collegio de Campolide, pp. 99-102; OLIVEIRA PINTO (1910b), O Instituto de Sciencias Naturaes do Collegio de Campolide». En O Nosso Colegio, 1908-1910, Lisboa, ed. del Collegio de Campolide, pp. 143-149.

106 Oliveira Pinto (1910a), pp. 99 y 104. 
«exsiccata e outras collecções já feitas ou assignará as que se estão distribuindo periodicamente» ${ }^{107}$. «Só este anno adquiriram-se mais de 1500 especies novas de fungos para o museu» ${ }^{108}$ «Procurará tambem augmentar essas colecções por meio de excursões scientificas como as que já se fizeram a Mafra, Gerez, Villa Viçosa, etc.; trocas com outros naturalistas, donativos feitos ao museu, etc.» ${ }^{109}$.

Se registraban intercambios de:

«fungos e musgos com naturalistas estrangeiros, principalmente com a Sociedade de Berlim, Berliner Botanische Tauschverein ${ }^{110}$.

Oliveira Pinto reconoce el papel fundamental de Torrend y Luisier en la «construcción» de estas preciosas colecciones de hongos y musgos del Colegio de Campolide:

«só a boa vontade e inquebrantável energia de trabalho dos meus dois collaboradores, P. A. Luisier e P. C. Torrend poderam conseguir o que se tem conseguido por meio do Instituto de sciencias naturaes do Collegio de Campolide» ${ }^{111}$.

¿Quiénes eran los recolectores del material para los herbarios de los Colegios? Torrend recogía hongos; Luisier principalmente musgos; Meniarth recogía plantas de las cercanías del Colegio de Torres Vedras, donde vivía; Valerio Cordeiro ${ }^{112}$, líquenes de la región de Setúbal ${ }^{113}$; Manuel Pacheco recogía algas y Sebastião Antunes, empleado del Herbario, también recogía plantas vasculares ${ }^{114}$.

107 Oliveira PinTo (1910a), p. 101.

108 OliVEIRA Pinto (1910b), p. 145.

109 Oliveira Pinto (1910a), p. 101.

110 Oliveira PinTo (1910b), p. 145.

111 Oliveira PinTo (1910b), p. 147.

112 Publica en Broteria un interesante trabajo de divulgación científica acerca de los líquenes: CORDEIRO, V.A. (1909), Os lichens. Que são e como se colleccionam?, Broteria, serie de Vulgarização Scientifica, VIII, pp. 75-80.

113 Publica un catálogo de los líquenes de Setúbal en Broteria, serie Botanica, XII, pp. 125-136, 177-192 y XIII, pp. 5-16.

114 TAVARES (1924). 
La implantación de la República en Portugal, el 5 de octubre de 1910, fue desastrosa para la actividad científica de los jesuitas botánicos y para la revista Broteria ${ }^{115}$. El 8 de octubre, un decreto «expulsa, desnaturaliza e espolia de tudo a todos os Jesuítas que residiam em Portugal» ${ }^{116}$.

En noviembre de 1910 fue nombrada una comisión para «estudarem o destino a dar aos livros e collecções de estudo do extincto Collegio de S. Fiel»»117. La comisión escribe una nota fechada el 22 de febrero de 1911, en la cual se proponía que el Herbario del Colegio fuese al «Liceu de Castelo Branco» ${ }^{118}$. Sin embargo, el gobierno decide contrariamente y manda entregar las colecciones de Historia Natural de S. Fiel a la Universidad de Coimbra' ${ }^{119}$. José Ramos Preto reacciona ante esta decisión, el gobierno modifica su plan y manda dividir el Herbario de S. Fiel entre el Liceu de Castelo Branco ${ }^{120}$ y el Herbario de la Universidad de Coimbra ${ }^{121}$.

La riqueza del Herbario de $\mathrm{S}$. Fiel en el momento de su desmembramiento fue descrita, pasados muchos años, por su director J.S. Tavares, en un trabajo publicado en 1924, en la propia revista Broteria:

«compunha-se de 93 espécies de Líchenes portugueses; 627 espécies de Musgos, portugueses e estrangeiros, incluindo a "Bryotheca Europaea" e a "Flora exsiccata Bavarica"; 521 espécies de Fungos portugueses; cêrca de 3.000 Diatomáceas, em que entravam as collecções compradas a Tempère e a Peragallo; 855 espécies e 106 subespécies de variedades de Phanerogâmicas portuguesas, a que se devem juntar 47 Phanerogâmicas estrangeiras. O número total das espécies de que se compunha o Herbário de $\mathrm{S}$. Fiel elevava-se por tanto a 5.121 espécies e o número de exemplares (não de espécies) de Phanerogamicas portuguesas ascendia a

115 L.G. Azevedo describió vivamente lo que ocurrió a los padres jesuitas en los días siguientes á la revolución republicana: Azevedo, L.G. (1911), Proscritos. Primeira parte, Valladolid, Florancio de Lara (ed.), 349 pp., disponible en http://purl.pt/12893 (consultado el 06/04/2010).

116 MENDES (1913), p. 9.

117 Mendes (1913), p. 28. El desmembramiento de las colecciones biológicas del Colegio de S. Fiel fue descrito por Salvado, M.A.N. (2001), O Colégio de S. Fiel - Centro difusor da Ciência no interior da Beira, Castelo Branco, Tip. Semedo y Salvado.

118 SALVADO (2001).

119 SALVAdo (2001).

120 Donde aún hoy se conserva, en la «Escola Secundária de Nuno Álvares».

121 SALVADO (2001). 
1.311, assim distribuídas: 27 Pteridófitas, 6 Gymnospérmicas, 231 Monocotyledóneas e 1.043 Dicotyledóneas» ${ }^{122}$.

En el Colegio de S. Fiel se encontraban las colecciones de diatomeas portuguesas. C. Zimmermann, exiliado en Brasil, describía con tristeza la pérdida de sus libros e instrumentos de laboratorio y las dificultades que había tenido para recuperar su colección de diatomeas y, naturalmente, la amargura de un naturalista que había sido expulsado del país que amaba:

«preparei uma grande collecção de diatomaceas de todas as regiões de Portugal e possessões ultramarinas, cujo estudo se estava publicando na revista Broteria, quando chegou a aurora da nova República Portugueza. (...) o governo (...) mandou restituirlhe, pelo menos, as collecções que com tanto trabalho reuniu, os instrumentos custosos do laboratório particular de micrographia, e a biblioteca de livros scientificos tão caros e tão necessários a um especialista. (...) a única cousa que esse governo me mandou restituir foi a minha collecção de diatomáceas e alguns manuscriptos. Nem isto teria feito, se um amigo generoso e dedicadíssimo se não interessasse por esta collecção. Lavro estas linhas como publico protesto contra o proceder injusto do governo provisório da Republica Portugueza para com um homem que, durante a sua estada em Portugal, teve sempre posta a mira em fazer bem ao paiz que lhe tinha dado hospitalidade» ${ }^{123}$.

También en noviembre fue nombrada, por el gobierno republicano, una comisión «encarregada de examinar urgentemente as colecções scientificas e a bibliotheca existentes no Collegio de Campolide, pertencente ao Estado, classificando o que encontrar digno de aproveitamento, e propondo ao Ministro da Justiça o destino a dar a esses objectos e livros, como entenderem mais util ao progresso da sciencia e ao enriquecimento das collecções, museus e bibliothecas de Lisboa» ${ }^{124}$. Esta comisión se reúne y vota por unanimidad que se restituyan las colecciones a sus legítimos dueños ${ }^{125}$. Pero gran parte de los libros, instrumentos y colecciones se habían perdido y sus dueños acabarían por recibir solamente una parte de lo que les pertenecía, y después de muchos meses de espera ${ }^{126}$.

En el Colegio de Campolide existía un sobresaliente herbario de hongos y una biblioteca de micología. C. Torrend, exiliado en Bélgica, describía en

122 TAVARES (1924), p. 83.

123 ZimmermanN, C. (1914), Catalogo das diatomáceas portuguesas, Broteria, serie Botanica, XII, pp. 115-124.

124 Mendes (1913), p. 16.

125 MeNDES (1913), p. 26.

126 Mendes (1913), pp. 26-27. 
Broteria las peripecias de sus estimadas colecciones que, finalmente, le serían restituidas $^{127}$

En respuesta a la usurpación, por parte el gobierno republicano, de las colecciones científicas de los colegios, los jesuitas escriben y divulgan un manifiesto fechado el 1 de julio de $1911^{128}$. El texto, escrito en portugués y francés, tuvo eco internacional, en concreto en la prestigiosa revista americana Science. En el número publicado el 24 de noviembre de 1911, T.D.A. Cockerell informaba a los lectores de lo que había pasado, y resumía un documento de protesta suscrito por los padres jesuitas exiliados ${ }^{129}$. Cockerell estaba de acuerdo en que los profesores jesuitas tenían derecho a recibir de vuelta sus bienes, libros y colecciones:

«granting the accusations of their enemies, that they are Jesuits, and (I suppose) opposed to a republican form of government, there is still no justification for the action taken in depriving them of their scientific materials. No doubt the government claims that all these things belong to the colleges, and not at all to the particular men; but while this may be true in a sense, all scientific men will agree that they had rights in the matter which have been apparently ignored» ${ }^{130}$.

A continuación, el autor sugería la formación de una comisión internacional para estudiar el asunto y elaborar un informe escrito:

«Would it be possible for some representative scientific body to appoint and pay the expenses of a man who would enquire into all the facts, and furnish a carefully considered report? Should such a plan be contemplated, preliminary enquires might be made to see whether the case of the exiles was apparently good. If the report supported the exiles, organized protests from the scientific bodies of different countries might be appropriate» ${ }^{131}$.

127 Torrend, C. (1912), Deuxième contribution pour l'étude des champignons de l'Ile de Madère, Broteria, serie Botanica, X, pp. 29-72.

128 TAVAreS, J.S. (1918), No comêço do quarto lustro da «Brotéria», Broteria, serie de Vulgarização Scientifica, XVI, pp. 5-11.

129 Cockerell, T.D.A. (1911), The exiled naturalists of Portugal, Science, 34, pp. 714-715.

130 COCKERELL (1911).

131 Cockerell (1911). 


\section{LOS JESUITAS DE BROTERIA EN EL EXTRANJERO Y GONÇALO SAMPAIO (1912-} 1922)

Expulsados de Portugal, los jesuitas de Broteria marchan el extranjero. Algunos van a Bélgica, se instalan en el Château de Dielighem, en Jette-Saint-Pierre, cerca de Bruselas, y fundan el Instituto Nun'Alvres que empieza sus clases el 7 de noviembre de $1912^{132}$. No obstante, la mayoría va directamente al Brasil. Después de algunos años, muchos regresan a la Península Ibérica y se reúnen en La Guardia, en el Colegio del Pasaje ${ }^{133}$, pero otros permanecerán en Brasil.

¿Qué sucedió con Broteria? Se interrumpe su publicación en 1910, pero reaparecerá en 1912, con el subtítulo de «Revista Luso-Brazileira». A pesar de las dificultades, las tres series de Broteria se mantienen publicándose en Salamanca (1913), en Tuy (1914), en Braga (1915-1923) y en Caminha después de 1924. Las tres series de Broteria diversifican los asuntos y colaboradores. La serie de divulgación científica cambia de secciones ${ }^{134}$.

Con la mayoría de los redactores viviendo en Brasil, Broteria publica muchos trabajos acerca de la historia natural de este país - su botánica, zoología, geología-, pero también acerca de la geografía y economía del Brasil. No obstante, continúa la publicación de los trabajos botánicos y zoológicos que habían sido realizados últimamente en Portugal. Algunos de los jesuitas portugueses ceden entonces lugar a los de otras nacionalidades, como P. ${ }^{\mathrm{e}} \mathrm{J}$. Foulquier ${ }^{135}$, profesor del Colegio P. ${ }^{\mathrm{e}}$ Antonio Vieira, en Baía ${ }^{136}$ y P. ${ }^{\mathrm{e}}$ Baltasar Merino, que vivía en el Colegio del Apóstol Santiago, en La Guardía ${ }^{137}$.

¿Qué trabajos son publicados en Broteria por sus fundadores, después de 1912 ?

132 La guerra con Alemania hará la permanencia del Colegio en Bélgica inviable. En 1914, el Instituto se muda al «Hotel de Los Placeres», en Lourizán, cerca de Pontevedra.

133 ONC (1914-1934), O Nosso Colégio, 1914-1934, edição do Instituto Nuno Álvares, Caldas da Saúde (Santo Tirso), 15 de Abril de 1934.

134 Las secciones son ahora: agricultura; animales utiles y nocivos; arboricultura; química; etnografía; geografía; comercio; historia de las ciencias; higiene; sismología; física; fisiología; medicina y variedades.

135 Que publica, en la serie de divulgación científica, varios trabajos pormenorizados acerca del caucho y del tabaco.

136 En este colegio vivían la mayoría de los redactores de Broteria.

137 B. Merino había publicado, una «Flora Descriptiva é Ilustrada de Galicia», en tres tomos (publicados en 1905, 1906 y 1909). En 1912, empieza la publicación, en Broteria, serie botánica, de unas «Adiciones a la Flora de Galicia». Estas adiciones serán publicadas en los tomos de 1912, 1913, 1914, 1915 y 1916. 
J.S. Tavares publica un catálogo de los insectos afideos portugueses ${ }^{138}$, continúa la publicación del estudio de las zoocecideas del continente portugués y Isla Madeira ${ }^{139}$ y empieza el estudio de las zoocecideas sudamericanas $^{140}$. En la serie de divulgación científica, publica un gran numero de trabajos acerca de temas botánicos, zoológicos, geográficos y económicos brasileños, como las plantas económicas (el café, el cacao, la caña de azúcar y el algodón); árboles frutales, animales salvajes, animales domésticos, importantes ciudades brasileñas o los sambaquís.

C. Zimmermann publica un apéndice al catálogo de las diatomeas portuguesas $^{141}$ y empieza el estudio de las diatomeas de Brasil ${ }^{142}$. Publica también un estudio preliminar de las diatomeas de Mozambique, recogidas por sus colegas P. ${ }^{\mathrm{e}}$ Luiz Lopes y P. ${ }^{\mathrm{e}}$ Manoel da Fonseca ${ }^{143}$.

C.A. Mendes continúa la publicación del estudio de los lepidópteros de Portugal ${ }^{144}$. Estudia también los de Galicia, Minho (con material recogido por Joaquim Duarte Roque, administrador de Broteria, exiliado en el Colegio de La Guardia ${ }^{145}$ ) y Salamanca ${ }^{146}$.

A. Luisier publica un estudio acerca de los musgos del genero Sphagnum de Brasil ${ }^{147}$.

A.C. Oliveira Pinto continúa la publicación de trabajos acerca de física y química.

C. Torrend continúa la publicación de su estudio acerca de los hongos (setas) de Isla Madeira ${ }^{148}$ y publica un estudio acerca de los hongos basidiomicetos de las cercanías de Lisboa y del Colegio de S. Fiel ${ }^{149}$. En 1913, inicia la edición de una exsicata de hongos (macromicetos) de Portugal y

138 Broteria, serie Zoologica, XII, pp. 177-193.

139 Broteria, serie Zoologica, XII, pp. 199-215 y XII, pp. 193-197.

140 Broteria, serie Zoologica, XIII, pp. 88-128, 145-160.

141 Broteria, serie Botanica, XII, pp. 115-124.

142 Broteria, serie Botanica, XI, pp. 149-164. Este primer trabajo acerca de las diatomeas de Brasil sería continuado en los años siguientes, en un extenso conjunto de trabajos: 1914 (XII, pp. 5-12), 1915 (XIII, pp. 37-56, 65-71, 124-146), 1916 (XIV, pp. 85-103, 130-157), 1917 (XV, pp. 30-45), 1918 (XVI, pp. 8-24, 113-122), 1919 (XVII, pp. 5-16).

143 Broteria, serie Botanica, XII, pp. 155-162.

144 Broteria, serie Zoologica, X, pp. 161-182 y XI, pp. 15-44.

145 Broteria, serie Zoologica, XII, pp. 61-75.

146 Broteria, serie Zoologica, XIII, pp. 55-61.

147 Broteria, serie Botanica, X, pp. 141-172.

148 Broteria, serie Botanica, X, pp. 29-49 y XI, pp. 165-181.

149 Broteria, serie Botanica, X, pp. 192-210 y XI, pp. 54-98. 
Brasil, «Fungi selecti exsiccati» ${ }^{150}$. En Brasil, estudia los mixomicetos ${ }^{151} \mathrm{y}$ los hongos parásitos del naranjo y del cacao.

L. Navás estudia los neurópteros sudamericanos ${ }^{152}$ y los líquenes de Isla Madeira ${ }^{153}$.

Con la marcha de muchos de los redactores de Broteria de Brasil a Galicia, la revista cambia nuevamente de asuntos $\mathrm{y}$, aunque muchos trabajos continúan discutiendo temas brasileños, los asuntos y colaboradores portugueses regresan a las páginas de la revista. G. Sampaio publica el primer trabajo en 1916.

J.S. Tavares continúa el estudio de las zoocecidias sudamericanas, en especial de Brasil ${ }^{154}$ y retoma el estudio de las ibéricas ${ }^{155}$. Publica, en la serie de divulgación científica, trabajos acerca de temas botánicos, zoológicos, geográficos, económicos y sociales de Portugal, como son la producción de vino y de corcho, la pesca en el río Mino, el analfabetismo, la criminalidad, los ferrocarriles, las caldas y las ciudades y regiones portuguesas.

C. Zimmermann continúa la publicación del catálogo de las diatomeas de Brasil ${ }^{156}$.

C.A. Mendes continúa el estudio de los lepidópteros de Salamanca ${ }^{157}$.

A. Luisier inicia la publicación, en 1917, de su monumental estudio acerca de los musgos de la Isla Madeira ${ }^{158}$, con material recogido por el prolífico botánico Carlos de Azevedo Menezes ${ }^{159}$.

C. Torrend publica un trabajo acerca de los hongos poliporáceos de Brasil $^{160}$. En la serie de divulgación científica, publica algunos trabajos

150 La exsicata estaba organizada en conjuntos de 100 pliegos. Los dos primeros conjuntos eran publicados en Broteria, serie botánica, tomos de 1913 (XI, pp. 99-104) y 1914 (XII, pp. 53-71). C. Torrend, viviendo en Brasil, anunciaba que Bresadola iba a colaborar en esta edición y que «la plupart des espèces seront récoltées dans le Nord du Brésil».

151 Publica un estudio preliminar en Broteria, serie Botanica, XIII, pp. 72-88.

152 Broteria, serie Zoologica, X, pp. 194-202; XI, pp. 45-53; XII, pp. 45-56, 215-234 y XIII, pp. 5-13.

153 Broteria, serie Botanica, X, pp. 73-100 y XI, pp. 5-32, 121-134.

154 En la serie zoológica, tomos XIV, XV, XVI, XVIII, XIX, XX y XXII.

155 En la serie zoológica, tomos XVI, XVII, XVIII, XIX, XX, XXI y XXII.

156 Broteria, serie Botanica, XIV, pp. 85-103, 130-157; XV, pp. 30-45; XVI, pp. 8-24, 113-122 y XVII, pp. 5-16.

157 Broteria, serie Zoologica, XVI, pp. 111-129.

158 Broteria, serie Botanica, XV, pp. 81-98; XVI, pp. 29-70; XVII, pp. 28-66, 112-142; XVIII, pp. 5-22, 79-120; XIX, pp. 36-48, 73-96 y XX, pp. 76-106.

159 Carlos de Azevedo Mendes publicará, en la serie botánica de 1922, 1926 y 1927, un conjunto de trabajos de grande valor acerca de la flora de Isla Madeira.

160 Broteria, serie Botanica, XVIII, pp. 23-43, 121-142; XX, pp. 107-112 y XXI, pp. 12-42. 
acerca de temas de la historia de la biología, como la generación espontánea, y un extenso relato de un viaje por el interior de Baía, Pernambuco y Ceará.

L. Navás continúa el estudio de los neurópteros sudamericanos ${ }^{161}$ e inicia la publicación del estudio de los tricópteros de España ${ }^{162}$.

Después de los años tumultuosos en el exilio brasileño, ya de vuelta a la Península Ibérica y viviendo en Pontevedra, J.S. Tavares escribe, en noviembre de 1917, uno importante texto acerca de los quince años de publicación de Broteria ${ }^{163}$. Sus primeras palabras, poéticas, son de un viajante que ha pasado tormentas, más que ahora reposa en lugar tranquilo:

«Ao defrontar-se com uma fonte amena, em tempo calmoso, o caminheiro não resiste a tomar algum allívio, ao murmúrio das águas. Já sentado á sombra do arvoredo, a fronte recostada no braço, relanceia então os olhos pelos montes e valles que percorreu, servindo-lhe de consolação o longo caminho andado, perante a perspectiva do que lhe falta ainda» ${ }^{164}$.

El viajante había caminado mucho, pero estaba aún lejos del fin del viaje:

«Tambêm nós jornadeamos há bons 15 annos, e distamos ainda da suspirada meta que nos propusemos. Ninguém extranhará, portanto, que, num momento de repoiso, depois de superadas as dificuldades de maior vulto, espraiemos a vista pelo caminho corrido nos três primeiros lustros da publicação da Broteria, pois esse desandar caminho com o pensamento não pode deixar de trazer-nos consolação, ânimo e ensinamentos» ${ }^{165}$.

Consolación, ánimo y voluntad, resumían el pasado ya vivido y el futuro que se aproximaba. La modesta, más intrépida y prevenida hormiga, era el ejemplo a seguir:

«Consolação, que não é pequena haver conservado uma revista scientífica de iniciativa particular, durante 15 annos, num meio tão restricto; ânimo para continuar, visto como difficilmente se nos atravessarão para o futuro obstáculos de maior monta do que os vencidos no último lustro; ensinamentos, enfim, porque o

161 Broteria, serie Zoologica, XIV, pp. 14-35.

162 Broteria, serie Zoologica, XIV, pp. 5-11, 139-146; XV, pp. 5-17, 63-68; XVI, pp. 7-20 y XVIII, pp. 23-27.

163 TAVARES (1918).

164 TAVARES (1918).

165 TAVARES (1918). 
modesto labor de três quinqénios mostra quanto pode a vontade decidida, ainda que desajudada e mesmo contrariada, ao modo da humilde formiga, que, esquecida de todos e moirejando dia e noite, vence mil empeços, emquanto vai encelleirando abundantes provisões que hão de sustentar a numerosa colónia em maus dias» ${ }^{166}$.

Con el exilio de los botánicos jesuitas no cesó el intercambio entre A. Luisier y J.S. Tavares con G. Sampaio. Al contrario, el período de permanencia en el Colegio del Pasaje fue incluso especialmente rico para el intercambio científico entre los botánicos jesuitas exiliados y G. Sampaio, y también para la calidad científica de Broteria.

Desde Salamanca, Luisier escribió a Sampaio congratulándose y ansiando por la visita de su colega de Oporto, denotando su continuada pasión por las briofitas y lamentándose de las pérdidas en los colegios jesuitas con la revolución republicana ${ }^{167}$.

J.S. Tavares escribe una carta a Sampaio desde Tuy; después de los años perturbados de lo pos-5-octubre-1910 y de su fuga a Brasil, ahora en suelo español y con condiciones para trabajar en historia natural, pensaba en el restablecimiento del intercambio científico con G. Sampaio. La pérdida de las colecciones zoológicas no ensonbrecía el animo. Su interés en las cecidias permanecía fuerte ${ }^{168}$.

En 1920 y 1921 Luisier y Tavares enseñan en el Colegio del Pasaje. Luisier prosigue sus estudios en briología. La publicación de Broteria y el Congreso de Oporto ${ }^{169}$ dominarán el intercambio epistolar entre Luisier, Tavares y Sampaio. Éste ultimo había publicado varios trabajos en la revista ${ }^{170}$. Era uno de los más eminentes botánicos peninsulares y por tanto su colaboración en Broteria contribuía al prestigio de la revista y, además, podría traer nuevos colaboradores.

166 TAVARES (1918).

167 Carta de A. Luisier a G. Sampaio, fechada el 28 de febrero de 1914, enviada de Salamanca (DB-FC-UP).

168 Carta de J.S. Tavares a G. Sampaio, fechada el 2 de enero de 1915, enviada de Tuy (DB-FC-UP).

169 El Congreso de Oporto (Oitavo Congreso de la «Asociación Española para el Progreso de las Ciencias», Oporto, 26 de junio-1 de julio de 1921) en el que G. Sampaio fue uno de los organizadores, se realizó conjuntamente con el Primer Congreso de la «Associação Portuguesa para o Progresso das Ciências».

170 Sampaio (1916a); Sampaio, G. (1916b), Centaurea Luisieri (sp. n.), Broteria, serie Botanica, XIV, pp. 104-105; SAMPAIO, G. (1917a), Líquenes novos para a flora portuguesa (2. ${ }^{a}$ série), Broteria, serie Botanica, XV, pp. 12-29; SAMPAIO, G. (1917b), Líquenes novos para a flora portuguesa (3. ${ }^{\mathrm{a}}$ série), Broteria, serie Botanica, XV, pp. 128-145. 
7. El REgRESO A PORTUGAL de LOS BOTÁNICOS JESUITAS Y LA CONSAGRACIÓN DE G. SAMPAIO

Después de algunos años de residencia en el Colegio de La Guardia, los jesuitas de Broteria regresan a Portugal, al Colegio Nuno Alvares, en Caldas da Saude, cerca de Santo Tirso, en el norte de Portugal ${ }^{171}$.

El reconocimiento público de la obra de G. Sampaio empieza en 1921 con la creación, en la Facultad de Ciencias de Oporto, de un instituto de investigación científica dedicado a la Botánica dirigido por el mismo, y culminará, en marzo de 1935, con la creación, en la Facultad de Ciencias de Oporto, del Instituto de Botânica Dr. Gonçalo Sampaio. Morirá dos años después.

\section{DISCUSIÓN Y CONCLUSIONES}

La revista Broteria recorrió un largo camino, desde su fundación en 1902, hasta mediados de la década de 1920. En el período inicial (1902-1907), publica casi solamente trabajos de sus fundadores sobre temas de investigación (entomología, micología, estudio de las diatomeas, briología) focalizados en el continente portugués. En 1907 amplía considerablemente su dimensión, temas y colaboradores. Naturalistas no jesuitas publican en Broteria. Los fundadores establecen una red de contactos y así estudian la historia natural de los territorios atlánticos y ultramarinos portugueses. La liquenologia es un nuevo tema. La serie de divulgación científica cumple un espacio vacío en la prensa periódica portuguesa.

La revolución republicana del 5 de octubre de 1910 tuvo un impacto inicial considerable en Broteria, pues la Compañía de Jesús fue expulsada de Portugal. Sin embargo, las dificultades del exilio parecen fortalecer y no enflaquecer Broteria, que se edita en 1912, manteniendo las tres series creadas en 1907. Los redactores en Brasil estudian la historia natural brasileña, y los que viven en Galicia, estudian la gallega. Nuevos colaboradores, como G. Sampaio, son invitados a publicar. Los Colegios jesuitas continúan en funcionamiento en el extranjero.

171 J.S. Tavares regresa a Portugal en 1928. Es electo socio de la Academia das Ciências de Lisboa. A. Luisier regresa a Portugal, en 1932. En el Colegio Nuno Alvares enseña, dirige el Museo de Ciencias Naturales, el Observatorio Meteorológico y Broteria. En 1933, es electo socio de la Academia das Ciencias de Lisboa. En 1942 es Doctor Honoris Causa en Ciencias Naturales por la Facultad de Ciencias de Oporto. Rebimbas Tavares regresa a Portugal, vive en Lisboa, Oporto e Covilhã. Cuando murió, era director del Instituto Superior de Filosofia en Braga. 
En la década de 1920, Broteria se establece como una de las mejores revistas de historia natural y una voz de la renovación de las Ciencias Naturales en Portugal. Para explicar el prestigio alcanzado podemos presentar los siguientes factores en sus veinte años de trabajo: las excepcionales calidades de sus fundadores y principales redactores: capacidad de trabajo, nivel intelectual y perseverancia; capacidad para establecer y mantener una red de contactos que les enviaban colecciones biológicas de regiones mal conocidas; capacidad de adaptación en el exilio, enfocando sus estudios en la historia natural del pais de exilio. Quizá, fueron esos mismos obstáculos del exilio los que demostraron y fortalecieron la excepcional capacidad de adaptación de los jesuitas de Broteria. La manutención, en regular funcionamiento, de sus colegios ( ¡la enseñanza es motor y muelle para la investigación!) y la apertura a naturalistas no jesuitas, naturalmente con otros modos de pensar y ver el mundo ( $i l a$ diversidad de temas enriquece una publicación generalista de ciencias naturales!) también deben haber contribuido al progreso de Broteria.

Los documentos epistolares discutidos en este trabajo demuestran la importancia de la construcción de redes de naturalistas interconectados, para el avance y desarrollo de las ciencias. Efectivamente, demostramos que A. Luisier, J.S. Tavares y G. Sampaio, lideraron una red de jesuitas naturalistas y botánicos de Oporto, que intercambiaron plantas e ideas. Este intercambio fue claramente importante para el estudio de la flora de musgos y plantas vasculares portuguesas y el progreso de los Herbarios de los colegios jesuitas y de la Academia Politécnica de Oporto. Quedó de manifiesto que estos progresos no resultaron de acciones aisladas de botánicos trabajando solos. También quedó clara la importancia de las fuentes documentales primarias (como son los documentos epistolares). Estos documentos son indispensables para intentar reconstruir al detalle el proceso de elaboración y avance de la ciencia botánica portuguesa en el inicio del siglo 20.

Fecha de recepción: 23 de enero de 2007

Fecha de aceptación: 31 de junio de 2008 